\title{
Relato de Experiência: Construção e Desenvolvimento do Programa de Saúde na Escola (PSE) sob a Perspectiva da Sexualidade na Adolescência
}

\author{
Case Study: Construction and Development of \\ the Health at School Program (PSE) from the \\ Perspective of Sexuality in Adolescence
}

Ana Carolina Drehmer Santosi Caroline Adriana Gasparim ${ }^{I}$ Gabriella Marques Monteiro Murilo Ribeiro Brito

Vanessa Alvez Mora da Silval

\section{PALAVRAS-CHAVE}

- Metodologia ativa.

- Medicina.

- Educação Sexual.

- Saúde Pública.

- Sistema Único de Saúde (SUS)

- Programa de Saúde na Escola (PSE).
O curso de Medicina da Universidade Federal do Pampa desenvolve-se sob pilares transformadores e vanguardistas da metodologia ativa. O curso transcende as práticas e educação biomedicalizadoras tradicionais por meio do reconhecimento dos aspectos humanos e sociais da medicina, em busca de uma formação centrada na visão holística do cuidado, preconizando um Serviço de Saúde público de qualidade. Além disso, a formação médica do Pampa traz à tona a necessidade de valorizar a autonomia do estudante, por meio de atividades que promovam a reflexão crítica e a aplicabilidade em situações reais dos temas propostos pelos docentes, valorizando ainda a articulação em grupo para diálogo, construção de ideias e resolução de problemas. A apropriação e aplicação de metodologias ativas em ambientes de promoção de saúde contribuem, portanto, para esse ideal de formação. Nessa perspectiva, este estudo consiste em um relato de experiência de abordagem crítico-reflexiva de acadêmicos do quarto semestre do curso de graduação em Medicina da Universidade Federal do Pampa (Unipampa). O estudo se refere ao componente de Práticas de Integração Ensino, Serviço e Comunidade (Piesc) do Núcleo de Prática Profissional (NPP) no período de abril a junho de 2018 e se trata de uma atividade proposta com o objetivo de compartilhar a vivência desses estudantes na construção e execução de uma atividade para o Programa de Saúde na Escola (PSE) vinculada a uma das Estratégias de Saúde da Família (ESF) de um município da fronteira oeste do Rio Grande do Sul. O assunto promovido pelos alunos foi escolhido por ser fundamental à prática de uma medicina com foco na prevenção de agravos e ainda assim pouco discutido devido à delicadeza do tema: saúde sexual. A atividade foi proposta com base teórica centrada no Aprendizado Baseado em Equipes (Team-Based Learning - TBL), destacando o autoconhecimento, prevenção contra infecções sexualmente transmissiveis, empoderamento no quesito de direitos sexuais e reprodutivos e o consentimento sexual. Foi realizada uma análise dialética das discussões com o propósito de avaliar as atividades realizadas, (re)significar as vivências e compilar as expectativas e impressões acerca da experiência. 


\section{KEY-WORDS}

- Active methodology.

- Medicine.

- Sex Education.

- Public Health.

- Unified Health System (SUS).

- Health at School Program (PSE).

\begin{abstract}
The Federal University of Pampa Medicine course is developed based on transformative, state-ofthe-art pillars of actioy methodology. The course transcends traditional practices and training geared toward biomedicalization by recognizing the social and human aspects of medicine, striving to provide a holistic care-based education, and advocating a public health service of quality. Furthermore, the UNIPAMPA medical school brings to the fore the need to value student autonomy, through activities that promote critical thinking and real-life applicability of the themes proposed by the faculty, highlighting the importance of group cooperation, dialogue, development of ideas and problem solving. The appropriation and applicability of active methodologies in health care contribute to this ideal of vocational training. The study therefore consists of an experience report of a criticalreflexive approach of fourth semester medical undergraduates from the Federal University of Pampa (UNIPAMPA). This study refers to the module of Teaching, Service and Community Intergration Practices administered at the Professional Practice Centre in the period from April to June 2018. The study aimed to share the students' experience in the building and execution an activity for the Health at School Program (PSE) tied to one of the Family Health Strategies (ESF) of a municipality on the western border of Rio Grande do Sul state. The topic developed by the students was selected as it represents an essential aspect of medical practice focused on preventing health issues and yet remains scarcely discussed due to the delicate nature of the theme: sexual health. The activity was proposed with a Team-Based Learning (TBL) theoretical basis, highlighting self-knowledge, prevention of sexual transmissible diseases, empowerment in the area of sexual rights and sexual consent. A dialectical analysis of the discussions was conducted in order to evaluate the activities, (re)define the experiences and compile expectations and impressions of the experiment.
\end{abstract}

Recebido em: 16/2/19

Aceito em: 30/3/19

\section{INTRODUÇÃO}

As políticas de saúde no Brasil são estruturadas em conformidade com princípios do Sistema Único de Saúde (SUS), e a atenção básica apresenta-se como uma estrutura fundamental à consolidação desse sistema de saúde. Assim, a partir da Constituição de 1988 surge também o SUS, caracterizado por ações de prevenção, promoção e proteção de saúde, as quais são desempenhadas por uma equipe multiprofissional que se dirige a populações de territórios bem delimitados. Segundo essa concepção, a atenção básica é tida como entrada principal dos usuários no sistema de saúde, valorizando a participação popular nos cuidados de saúde. Ainda, a Estratégia de Saúde da Família (ESF) é um meio de qualificar e consolidar a atenção básica, almejando uma expansão dos impactos positivos na situação de saúde dos indivíduos e sociedade ${ }^{1}$.

Nesse cenário, com base em análises da Política Nacional de Atenção Básica (Pnab), entende-se que o profissional médico tem um papel fundamental junto à equipe multiprofissional - a qual deve contar com, no mínimo, médico, enfermeiro, auxiliar ou técnico de enfermagem e agentes comunitários de saúde. Além da prática de consultas clínicas e pequenos pro- cedimentos cirúrgicos, são de responsabilidade médica atividades em grupo na UBS e nos demais espaços comunitários, como escolas e associações.

Compreendendo a escola como um espaço potencial para ampliar e garantir o alcance do SUS, o PSE foi instituído em 2007 a fim de colaborar, mediante ações de prevenção e promoção de saúde, para a formação integral dos estudantes da rede pública de educação básica. Nesse sentido, é uma proposta dos ministérios da Saúde e da Educação, que atua também em vulnerabilidades que possam comprometer o desenvolvimento escolar dos estudantes.

Nessa perspectiva, os novos cursos de Medicina no Brasil vêm propondo inovação na formação médica brasileira em consonância com as propostas e princípios do SUS, bem como com seus programas. O curso de Medicina da Unipampa, aberto pelo Programa Mais Médicos, faz parte desse grupo de cursos criados com tal propósito. Foi inaugurado em 2016, e nele, desde o primeiro semestre, os alunos têm contato com amplas teorias para compreender o próprio funcionamento do curso: a metodologia ativa, além da promoção da integração da formação acadêmica com os serviços de saúde públicos ${ }^{2}$. 
Segundo a proposta acadêmica do componente de Práticas de Integração Ensino, Serviço e Comunidade (Piesc) do Núcleo de Prática Profissional (NPP) do curso de Medicina da Unipampa, os acadêmicos do quarto semestre da graduação se inserem na realidade da atenção básica municipal, com ênfase nas ações de educação em saúde. O objetivo deste trabalho é relatar a vivência destes estudantes no PSE, ressaltando a contribuição da formação acadêmica embasada em metodologias ativas para a construção e execução das atividades de inserção prática.

\section{METODOLOGIA}

Trata-se de um relato de experiência, de abordagem crítico-reflexiva, acerca da vivência de acadêmicos do quarto semestre da graduação em Medicina da Unipampa, por meio da aplicação de metodologias ativas em uma ação do PSE. Tal experiência ocorreu durante as atividades práticas do componente curricular do Piesc. Este componente objetiva incentivar os discentes a realizar ações de educação em saúde com a população que utiliza os serviços da atenção básica.

A metodologia escolhida para a análise deste relato foi a Metodologia da Problematização por meio do Arco de Maguerez, devido à necessidade observada de ampliar a visão crítica sobre a realidade relativa à saúde sexual. Os estudantes de Medicina assumiram o papel de tutor, guiando os alunos da escola por meio de um caminho didático. Primeiramente, os tutores se apresentaram, expondo o tema para trazer a realidade concreta do assunto tratado. A reflexão sobre a realidade concreta se iniciou por meio da resolução de um teste de 20 perguntas pelos alunos. Foram 30 minutos para discussão em pequenos grupos de 10 a 20 pessoas. As perguntas se referiam a anatomia, fisiologia, infecções sexualmente transmissíveis e direitos reprodutivos e sexuais, entre outros tabus sociais sobre a sexualidade. A composição das perguntas objetivou gerar uma reflexão crítica e problematização a partir de conhecimentos prévios dos alunos. O caminho didático continuou por meio da resolução das mesmas questões em grande grupo, mediante o estabelecimento de pontos-chave e exposição teórica dos temas, contando com grande participação devido ao confronto com ideias preestabelecidas e juízos. A síntese, então, foi a desconstrução de muitas opiniões dos estudantes, tanto os de idade escolar quanto os acadêmicos, e uma reconstrução pautada em conhecimentos embasados filosófica e biologicamente via reflexão crítica ${ }^{3}$.

A vivência ocorreu em abril de 2018, em uma Estratégia de Saúde da Família (ESF) de um município da fronteira oeste do Rio Grande do Sul, em um turno matutino e um vespertino. Essa ESF é composta por uma equipe multiprofissional que conta com uma dentista, uma auxiliar de dentista, um educador físico, uma médica, uma enfermeira, duas técnicas em enfermagem, uma recepcionista e cinco agentes comunitários. Essa equipe é responsável por uma população de aproximadamente 4.700 pessoas e conta com cinco microáreas descobertas. Durante a experiência relatada, a ESF foi campo de estudo semestral dos acadêmicos da Unipampa.

A partir da proposta do componente curricular Piesc IV, os acadêmicos se responsabilizaram pelo tema Direito Sexual e Reprodutivo (planejamento reprodutivo, dupla proteção prevenção da gravidez na adolescência - roda de conversa) e prevenção de DST/aids, indicado pelo calendário da Secretaria de Saúde do município. Os acadêmicos contaram com o apoio da médica responsável pela ESF em questão - que atuou como preceptora dos acadêmicos na unidade básica de saúde - em todas as etapas do processo que culminaram na experiência relatada. Para efetuar o programa, os alunos consultaram materiais disponibilizados pelo Ministério da Saúde, em parceria com o Ministério da Educação, que sugerem atividades e discussões sobre o tema, adaptando-os. As didáticas utilizadas foram adequadas a partir das construções diárias da faculdade de Medicina, isto é, houve uma adaptação da metodologia Team-Based Learning (TBL) à realidade na qual os alunos se inseriram.

Após a execução das atividades práticas, os estudantes de graduação e a médica preceptora participaram de uma discussão a fim de avaliar as atividades, (re)significar as vivências e compilar as expectativas e impressões acerca da experiência.

Com a finalidade de discutir a vivência dos acadêmicos, serão apresentados a seguir os resultados e uma discussão dos mesmos com fundamentação teórica.

\section{RESULTADOS E DISCUSSÃO}

A escola em que foram realizadas as atividades se situa na área de abrangência da ESF apresentada anteriormente. Participaram da didática 112 alunos, com presença homogênea em relação ao gênero. A participação efetiva e prática nas atividades também se dividiu de forma homogênea entre os gêneros, demonstrando a importância e o interesse do tema saúde sexual nesse cenário.

Pode-se notar que as idades dos alunos foram variadas devido ao grande número de turmas que se fizeram presentes - no total, três turmas participaram das atividades propostas. O predomínio, no entanto, foi de alunos com mais de 15 anos, idade importante para o tema proposto, devido à iniciação em atividades sexuais.

A importância do trabalho sobre o desenvolvimento da sexualidade e conteúdos relativos ao conhecimento biológi- 
co está embasada em uma medicina preventiva, preconizada pelo SUS. O conhecimento sobre doenças e sua prevenção, métodos contraceptivos, empoderamento e direitos sexuais e reprodutivos, além de autoconhecimento sobre as transformações corpóreas fisiológicas e psicológicas que ocorrem na fase da adolescência, formulado pela reflexão crítica e com acesso à discussão com conhecimentos embasados, tem um grande poder para evitar futuras situações de risco ${ }^{4}$.

Segundo relatório divulgado pela Organização Mundial da Saúde (OMS) em abril de 2018, a taxa de adolescentes grávidas é de cerca de 68,4 nascimentos para cada mil meninas, uma porcentagem alarmante que supera a taxa sul-americana de 65,5 nascimentos e está muito além da taxa mundial de 46 nascimentos para cada mil meninas de acordo com a Pan American Health Organization, United Nations Population Fun e United Nations Children's Fund ${ }^{5}$. Além disso, a recorrência de IST é preocupante dentro do assunto da sexualidade. A cidade de Porto Alegre, por exemplo, ocupa a segunda posição no ranking geral; já Uruguaiana, com população superior a 100 mil habitantes, ocupa a quinta posição geral em relação ao índice de infecção por aids/HIV, segundo a Secretaria da Saúde do Rio Grande do Sul' .

Laraia $^{7}$ discute a importância da cultura na formação de comportamentos sociais e até mesmo de posturas corporais em função do sexo. Assim, segundo a compreensão de que a visão de mundo é o resultado da operação de determinada cultura, os acadêmicos buscaram compreender tal aspecto para construir a didática aplicada no PSE. As atividades foram pensadas de forma que fossem significativas e relevantes naquele contexto cultural, ponderando linguagem oral, escrita e visual, bem como os recursos utilizados.

\section{Formação médica, metodologia e a adaptação ao PSE}

O processo educacional moderno resulta da constante evolução de ideias, que permeia diversos pensadores - iniciado por Montessori, com a aprendizagem pelo condicionamento, seguida de Frenet, com a aprendizagem por meio da experiência, Piaget e Vygotsky. Sob a mesma linha teórica, a estratégia de aprendizagem pelo Team-Based Learning ${ }^{8}$, desenvolvido por Larry Michaelsen et al. ${ }^{9}$ nos anos 2002, proporciona aos alunos a oportunidade de desenvolver assuntos de maneira independente, no sentido da relação dupla estabelecida entre professor e aluno, na qual a coparticipação na construção do conhecimento é essencial ao sucesso do aprendizado significativo ${ }^{10}$.

Nessa perspectiva, o TBL estimula os acadêmicos ao trabalho em equipe, visualizando a solução de casos clínicos reais, e, conforme Burgess ${ }^{11}$, desenvolve nos participantes dessa dinâmica um senso de preocupação e responsabilidade para com os colegas de grupo. A aplicação dos conhecimentos à solução de um problema prático ainda consolida tais conhecimentos e os torna mais palpáveis e simbólicos. A proposta de educação médica visa ampliar a autonomia do estudante e modificar o modelo hierárquico vertical de educação, trazendo os estudantes para um estudo contextualizado e significativo.

Segundo a concepção de TBL, a metodologia foi adaptada para a vivência do PSE. Os alunos de Medicina atuaram como facilitadores do processo de ensino-aprendizagem, respeitando a autonomia dos estudantes da escola quanto ao direcionamento da atividade às questões que levantam mais dúvidas entre eles, a fim de desenvolver nos participantes o senso de preocupação com o conhecimento a ser desenvolvido e a responsabilidade, além de proporcionar um trabalho contextualizado e dinâmico em equipes.

Inicialmente, os acadêmicos se apresentaram e expuseram o TBL e a metodologia ativa por meio de palavras fáceis e "memes" (imagens engraçadas da internet). Então, direcionaram os estudantes da escola para a formação de grandes grupos, que receberam um material com perguntas relacionadas ao tema proposto - 15 questões a serem resolvidas em grupos em dez minutos. Em seguida, entregaram uma placa de papel a cada grupo que havia se formado com opções de A até D e uma placa com "concordo" e outra com "discordo" para que os grupos pudessem interagir com o feedback imediato que a metodologia propõe.

Dessa maneira, a não diretividade atua como fator sedimentador de uma construção embasada na autonomia do aluno participativo e no potencial de um ambiente democrático ${ }^{12}$. Após um tempo para discussão e resposta, os graduandos utilizaram a projeção de slides para fornecer feedback aos alunos, discutindo todos juntos as perguntas, explicando um pouco sobre anatomia feminina e masculina, métodos contraceptivos e IST - enfatizando o HPV, visto que na semana seguinte ocorreria uma campanha de vacinação na mesma escola.

Após esse feedback, foram surgindo dúvidas por parte dos alunos da educação básica, que foram respondidas e debatidas em conjunto por acadêmicos, médica e alunos. Por fim, foram apresentadas situações reais que poderiam exigir as habilidades e conteúdos desenvolvidos naquele espaço, contando com a participação ativa dos alunos. Assim, foi possível observar a motivação dos alunos ao responderem e "corrigirem" logo em seguida suas respostas, bem como sua disposição em aplicar os conhecimentos no final da atividade.

Além disso, os estudantes trataram de empoderamento, consentimento, direitos sexuais e tabus relativos a relações sexuais. Iniciaram o assunto com um vídeo que compara o ato 
sexual ao ato de tomar chá, explicando de forma simples e óbvia o consentimento sexual pelo Blue Seat Studios ${ }^{13}$. Abordaram ainda homossexualidade e a importância do uso de camisinha em todas as relações, ressaltando que o HIV não é uma doença exclusiva de relações homoafetivas.

Os assuntos inicialmente pareciam básicos, mas ao longo das atividades os alunos notaram a importância de falar sobre assuntos fundamentais e de forma divertida e integrada. Cada aluno, com sua história e influências, ouvia atento, confiando nas informações que estavam em discussão. A participação foi tão intensa que na última turma os alunos contaram com a interação de um professor que colaborou com eles para responder às perguntas propostas. A atividade final convidava os alunos a virem à frente do grupo para responderem a perguntas relacionadas ao tema. Houve várias respostas engraçadas e decididas. Uma das meninas, à pergunta "Se um menino te diz: 'Eu morro, mas não uso camisinha!', o que você diz para ele?", respondeu: "Então morre, diabo!". Outro aluno foi indagado: "Se uma menina te disser: 'não vamos usar camisinha, apenas homossexuais têm HIV', você responde o quê?". A aluna respondeu que estava correto, mas usaria camisinha porque havia também o risco de gravidez e outras infecções sexualmente transmissíveis. As respostas errôneas foram discutidas novamente a fim de consolidar os conceitos abordados.

Nesse sentido, fica evidente que o trabalho de promoção de saúde com os alunos deve almejar desenvolver em cada um a capacidade de interpretar o dia a dia e atuar de maneira comprometida para a melhoria da qualidade de vida mediante atitudes e comportamentos. Logo, os profissionais de saúde se responsabilizam pelo empoderamento de estudantes, professores e funcionários das escolas, estabelecendo um vínculo que possibilite o acesso em futuras necessidades das crianças e adolescentes.

\section{CONSIDERAÇÕES FINAIS}

Diante do processo de construção da vivência, execução e reflexão, é possível observar que tais atividades induzem o acadêmico a exercer seu papel no processo de construção de conhecimento, alinhando conhecimentos teóricos - que obrigatoriamente se contextualizam e ganham novos significados - à prática. Logo, fica evidente que os objetivos de troca, desconstrução e reconstrução de conceitos que permeavam a promoção de saúde proposta pelos acadêmicos foram alcançados com êxito por meio da formação acadêmica baseada em metodologias ativas que possibilitam o desenvolvimento amplo do aluno. Nesse sentido, o aluno da formação moderna desenvolve habilidades comunicativas, autonomia, responsabilidade e segurança para liderar e administrar atividades de promoção de saúde a partir de sua inserção precoce na atenção básica.

Analisando os reflexos da atuação no PSE na formação médica, percebe-se que o estudante, diante desse contexto, consegue enxergar a importância de ações de promoção de saúde, uma vez que visualiza o amplo alcance de ações como as desenvolvidas por meio desses programas e o impacto que podem trazer para a saúde da comunidade.

Destaca-se também a relevância do trabalho multidisciplinar e interdisciplinar, em equipe e também intersetorial, aliando saúde e educação, duas áreas responsáveis pelas mudanças profundas e de base na sociedade. Da mesma forma, reitera-se o compromisso do curso de Medicina da Unipampa em promover uma devolutiva à população no âmbito das competências que tangem saúde e sociedade.

\section{REFERÊNCIAS}

1. Rolim, L. B.; Cruz, R. S. B. L. C; Sampaio, K. J. A. J. Participação popular e o controle social como diretriz do SUS: uma revisão narrativa. Capturado em 18 de novembro, 2018. Revista Saúde em Debate - Rio de Janeiro, v. 37, n. 96, p. 139-147, jan./mar. 2013.

2. Farias PAM, Martin ALAR, Cristo CS. Aprendizagem Ativa na Educação em Saúde: Percurso Histórico e Aplicações. Revista Brasileira de Educação Médica 2015; 39(1): 143-150.

3. Caldeira MAS, Zaidan S. Práxis pedagógica: Um desafio cotidiano. Revista Paidéia 2013; 14:15-32.

4. Saito, M. I.; Leal, M. M. Educação sexual na escola. Instituto da Criança do Hospital das Clínicas e Departamento de Pediatria da Faculdade de Medicina da Universidade de São Paulo. 2000, 22(1): 44-48. Capturado em 17 de novembro, 2018. Disponível em: < https://s3.amazonaws. com/academia.edu.documents/39242838/Educacao_sexual_na_escola.pdf?AWSAccessKeyId=AKIAIWOWYYG Z2Y53UL3A\&Expires $=1542722475 \&$ Signature $=\% 2 B a z V 5 r$ 0DORd4adm8KU0zjafrKBs\%3D\&response-content-dispo sition=inline \%3B\%20filename\%3DEducacao_sexual_na_ escola.pdf $>$

5. Pan American Health Organization, United Nations Population Fund, and United Nations Children's Fund. Accelerating progress toward the reduction of adolescent pregnancy in Latin America and the Caribbean; 2017. Capturado em 14 de novembro de 2018. Disponível em: <http://iris.paho.org/xmlui/bitstream / handle / 123456789 /34493/9789275119761-eng. pdf? sequence $=1 \&$ isAllowed $=y \& u a=1>$

6. Secretaria da Saúde do Rio Grande do Sul. Departamento de ações em saúde. Coordenação Estadual de IST/AIDS. 
Situação da Epidemia de HIV / Aids no Rio Grande do Sul; 2017. Capturado em 14 de novembro de 2018. Disponível em: <https://atencaobasica.saude.rs.gov.br/upload/ arquivos /201712/05161516-situacao-da-epidemia-de-hiv-aids-2017.pdf>

7. Laraia RB. Cultura: um conceito antropológico. Rio de Janeiro: Editor Jorde Zahar; 2001

8. Bolella VR, Senger MH, Tourinho FSV, Amaral E. Aprendizagem baseada em equipes: da teoria à prática da teoria à prática. Medicina 2014; 47(3): 293-300.

9. Michaelsen L, Knight A, Fink L. Team-based learning: a transformative use of small groups. New York: Stylus; 2002.

10. Mitre SM, Batista RS, Mendonça JMG, Pinto NMM, Meirelles CAB, Porto CP, Moreira T, Hoffmann, LMA. Metodologias ativas de ensino-aprendizagem na formação profissional em saúde: debates atuais. Ciência \& Saúde Coletiva 2008; 13(2): 2133-2144.

11. Burguess, AW. Applying Established Guidelines to Team-Based Learning Programs in Medical Schools: A Systematic Review. BMC Academic Medicine: April 2014, Volume 89, Issue 4 - p 678-688. Capturado em 25 de junho, 2018. Disponível em: <https://journals.lww.com/academicmedicine/Fulltext/2014/04000/Applying_Established_Guidelines_to_Team_Based.38.aspx>.

12. Brooks W. Team Based Learning in Medical Education. University of Alabama at Birmingham. Capturado em 24 de junho, 2018. Disponível em: <https://www.uab.edu/ medicine/gim/images/Team-based_learning_TBL_in_ Med_Ed.pdf>.

13. May, E.; Blue Seat Studios. Tea Consent. 2015. (2m51s). Capturado em 25 de setembro, 2018. Disponível em: $<$ https:/ / www.youtube.com/watch?v=oQbei5JGiT8>.

\section{CONTRIBUIÇÃO DOS AUTORES}

Os autores abaixo relacionados contribuíram efetivamente para a construção do Relato de Caso Relato de Experiência: Construção e Desenvolvimento do Programa de Saúde na Escola (PSE) sob a Perspectiva da Sexualidade na Adolescência nas seguintes funções:

1) Construção da atividade com pesquisa em base de dados e protocolos para realização da atividade no Programa Saúde na Escola:

Ana Carolina Drehmer Santos

Caroline Adriana Gasparim

Gabriella Marques Monteiro

Murilo Ribeiro Brito
Vanessa Alvez Mora da Silva

2) Prática da atividade para os adolescentes no Programa Saúde na Escola:

Ana Carolina Drehmer Santos

Caroline Adriana Gasparim

Gabriella Marques Monteiro

3) Redação do Relato:

Ana Carolina Drehmer Santos

Caroline Adriana Gasparim

Gabriella Marques Monteiro

Murilo Ribeiro Brito

4) Padronização do texto segundo normas:

Ana Carolina Drehmer Santos

Caroline Adriana Gasparim

Gabriella Marques Monteiro

5) Revisão do texto e correções:

Ana Carolina Drehmer Santos

Caroline Adriana Gasparim

Gabriella Marques Monteiro

\section{CONFLITO DE INTERESSES}

Ao Editor Científico da Revista Brasileira de Educação Médica

\section{DECLARAÇÃO DE CONFLITOS DE INTERESSE}

Nós, Ana Carolina Drehmer Santos, Caroline Adriana Gasparim, Gabriella Marques Monteiro, Murilo Ribeiro Brito e Vanessa Alvez Mora da Silva, autores do Relato de Caso intitulado "Relato de Experiência: Construção e Desenvolvimento do Programa de Saúde na Escola (PSE) sob a Perspectiva da Sexualidade na Adolescência" declaramos que nos últimos 5 anos e nos futuros próximos não possuímos conflito de interesse de ordem:

(X) financeiro,

(X) comercial,

(X) político,

$(X)$ acadêmico e

(X) pessoal,

Declaramos também que o apoio financeiro e (ou) material recebido para o desenvolvimento deste trabalho estão claramente informados no texto.

As relações de qualquer tipo que possam levar a conflito de interesse estão completamente manifestadas abaixo.

Uruguaiana, Sexta-Feira, 26 de Julho de 2019. 


\section{ENDEREÇO PARA CORRESPONDÊNCIA}

Universidade Federal do Pampa - UNIPAMPA

BR 472 - Km 585 - Caixa Postal 118 - Uruguaiana, RS

CEP $97501-970$ 